\title{
NOUVELLE
}

\section{$+$ \\ Un nouveau mécanisme de contrôle de la douleur : DREAM ou réalité ?}

François Cesselin
Service de Biochimie et de Biologie Moléculaire, Inserm U.288, Faculté de Médecine Pitié-Salpêtrière, 91, boulevard de l'Hôpital, 75013 Paris, France.
$>$ Les peptides opioïdes endogènes et leurs récepteurs sont impliqués dans de nombreuses fonctions essentielles pour la survie des individus et des espèces, notamment la douleur. Trois principaux récepteurs des opioïdes ( $\mu, \delta$ et $\kappa)$ ont été clonés et leurs gènes séquencés. Leurs ligands endogènes sont constitués d'une vingtaine de peptides qui appartiennent à trois grandes familles selon le précurseur dont ils proviennent. La proopiomélanocortine (POMC) est à l'origine de la $\beta$-endorphine et de peptides apparentés ; la pro-enképhaline est source des enképhalines et de peptides voisins; enfin, la pro-dynorphine donne naissance aux néo-endorphines et aux dynorphines [1]. Deux autres peptides opioïdes (endomorphine-l et endomorphine-2), dont le précurseur n'est pas encore identifié, ont été découverts plus récemment [2]. C'est dans la corne dorsale de la moelle épinière que sont situés les premiers relais des « voies de la douleur » et que le contrôle de la douleur par les opioïdes est le moins mal connu (Figure 1). On trouve dans cette région des terminaisons enképhalinergiques et dynorphinergiques, appartenant pour la plupart à des interneurones spinaux. Ceux-ci modulent la transmission des messages nociceptifs par le biais de la liaison des peptides opioïdes aux récepteurs $\mu, \delta$ et $\kappa[3]$. La morphine et les autres opioïdes exogènes exercent leurs nombreuses actions pharmacologiques, en particulier leurs effets analgésiques, par l'intermédiaire de ces mêmes récepteurs.

La transcription du gène de la prodynorphine est contrôlée par divers fac- teurs agissant notamment sur des séquences de type CRE ( $C A M P$-responsive element) et une séquence plus récemment identifiée, appelée DRE (pour downstream regulatory element) [4]. Une protéine, appelée DREAM (DREantagonistic modulator), contenant quatre domaines de type $\varepsilon F$ hand (présents fréquemment dans les molécules se liant au $\left.\mathrm{Ca}^{2+}\right)$, se fixe à la séquence $D R \varepsilon$ pour réprimer l'expression du gène. Sous l'influence du $\mathrm{Ca}^{2+}$, DREAM perd sa capacité de liaison à l'ADN et l'inhibition de la transcription du gène est levée [5]. DREAM est le premier facteur de régulation de la transcription directement contrôlé par le $\mathrm{Ca}^{2+}$ qui ait été isolé.

Dans un article récent, Cheng et al. [6] montrent que l'invalidation du gène codant pour la protéine DREAM chez la souris conduit à une réduction des réponses à diverses stimulations douloureuses, sans affecter d'autres fonctions (motricité, mémoire, immunité...). Cette réduction est attribuée à une augmentation de la transcription du gène codant pour la pro-dynorphine dans la moelle épinière. De fait, l'hyposensibilité aux stimulations nociceptives des animaux dépourvus de la protéine DREAM est totalement, ou partiellement, abolie par le blocage des récepteurs des opioïdes, en particulier des récepteurs $\kappa$ dont les dynorphines sont probablement les ligands endogènes [7]. De plus, on note une augmentation du taux des ARNm codant pour la pro-dynorphine, et des concentrations d'une des formes de dynorphine, dans des extraits de moelle épinière d'animaux dont le gène codant pour DREAM est inactivé. De manière sur- prenante, la transcription du gène codant pour la prodynorphine dans d'autres régions n'apparaît en rien affectée par l'absence de la protéine DREAM. Le contrôle normalement exercé par la protéine DREAM semble particulièrement sélectif puisque, de surcroît, l'expression des autres gènes dotés d'une séquence DRE (gènes codant pour la pro-enképhaline et pour la protéine c-Fos) reste inchangée chez les souris mutées. En revanche, dans des cellules transfectées, l'expression du gène $c$-fos est clairement dépendante de DREAM [5]. Ainsi, la voie de régulation de l'expression du gène codant pour la pro-dynorphine, qui implique la protéine c-Fos via un site de type AP-1 (activator protein-1), et qui est connue depuis longtemps [8], n'apparaît pas sollicitée pour accroître la synthèse du précurseur pro-dynorphine. Ces conclusions reposent sur l'analyse d'images obtenues après hybridation de riboprobes sur des sections de moelle épinière. L'une de ces images, qui ne fait l'objet d'aucun commentaire de la part des auteurs, est tout à fait étrange. Elle montre en effet que I'ARNm le plus abondant, chez la souris témoin comme la souris mutée, est celui de la POMC. Cette découverte, si elle est confirmée, est d'importance. De fait, chez le rat en tout cas, la majorité des auteurs s'accordent pour conclure que la quasi-totalité des peptides dérivés de ce précurseur POMC présents dans la moelle épinière ne sont pas synthétisés localement, mais sont contenus dans les terminaisons de neurones d'origine supra-spinale [9].

Les résultats publiés par Cheng et al. [6] sont troublants à un autre titre. En effet, que les dynorphines exercent une action spinale anti-nociceptive est une conclusion qui va totalement à l'encontre de tout un ensemble de données convergentes. Jusqu'à présent, on s'accordait 
généralement pour considérer que, si les enképhalines réduisent la transmission spinale de la douleur (effet anti-nociceptif), les dynorphines, en revanche, la facilitent (effet pro-nociceptif) [10, 11]. Par exemple, des études détaillées réalisées chez des souris dépourvues du gène codant pour la pro-dynorphine montrent très clairement que les dynorphines spinales exercent des effets pronociceptifs et jouent un rôle majeur dans le maintien des douleurs liées à des lésions nerveuses (douleurs neuropathiques) [11]. Or, les douleurs de ce type sont réduites chez les souris dont le gène codant pour DREAM est inactivé [6].

Des résultats aussi contradictoires sont peut-être tout simplement dus aux possibles biais de l'approche consistant à invalider un gène. Les anomalies phénotypiques observées chez des souris porteuses d'un gène invalidé peuvent, par exemple, résulter des effets du fond génotypique [12]. Une explication moins triviale est proposée par Cheng et al. [6] : selon leurs concentrations extracellulaires, et les récepteurs qu'elles sollicitent (récepteurs $\kappa$ ou une catégorie de récepteurs des acides aminés excitateurs, les récepteurs NMDA [N-méthyl-D-aspartate]) [10], les dynorphines exerceraient soit des effets anti-nociceptifs soit, au contraire, des effets pro-nociceptifs. Une autre hypothèse peut être évoquée. Elle tient compte des propriétés de DREAM, dont l'action ne se limite pas à celle d'un répresseur de la transcription, comme l'indiquent les autres noms qui lui ont été attribués (calséniline [13] ou KChIP3 [14]). C'est ainsi que DREAM se lie à des canaux potassiques sensibles au potentiel de type $A$ et en règle l'activité d'une manière qui dépend des fluctuations de la concentration de $\mathrm{Ca}^{2+}$ intracellulaire (d'où le nom de KChIP: $K$ channel-interacting protein) [14]. Certes, les souris n'exprimant pas DREAM ne présentent aucune anomalie cardiaque décelable bien que des canaux ioniques de ce type (Kv4) aient été impliqués dans des pathologies car- diaques. Les neurones sensoriels possèdent de tels canaux, appartenant notamment au sous-type Kv4.2, qui jouent un rôle important dans le contrôle de leur excitabilité [15]. L'expression très précoce du gène de Kv4.2 chez la souris suggère que cette protéine est impliquée dans le développement [16]. Ainsi, l'absence de la protéine DREAM pourrait bien affecter la sensibilité des neurones sensoriels aux stimulations nociceptives surtout si leur développement embryonnaire est altéré. Ces changements pourraient intervenir dans les modifications de la sensibilité à la douleur des souris mutées étudiées par Cheng et al. [6] L'augmentation sélective de l'expression du gène de la pro-dynorphine dans la moelle épinière pourrait même alors être interprétée comme résultant d'un phénomène compensatoire.

En conclusion, les observations rapportées par Cheng et al. [6] posent, à nouveau, la question du rôle réel des dynor-

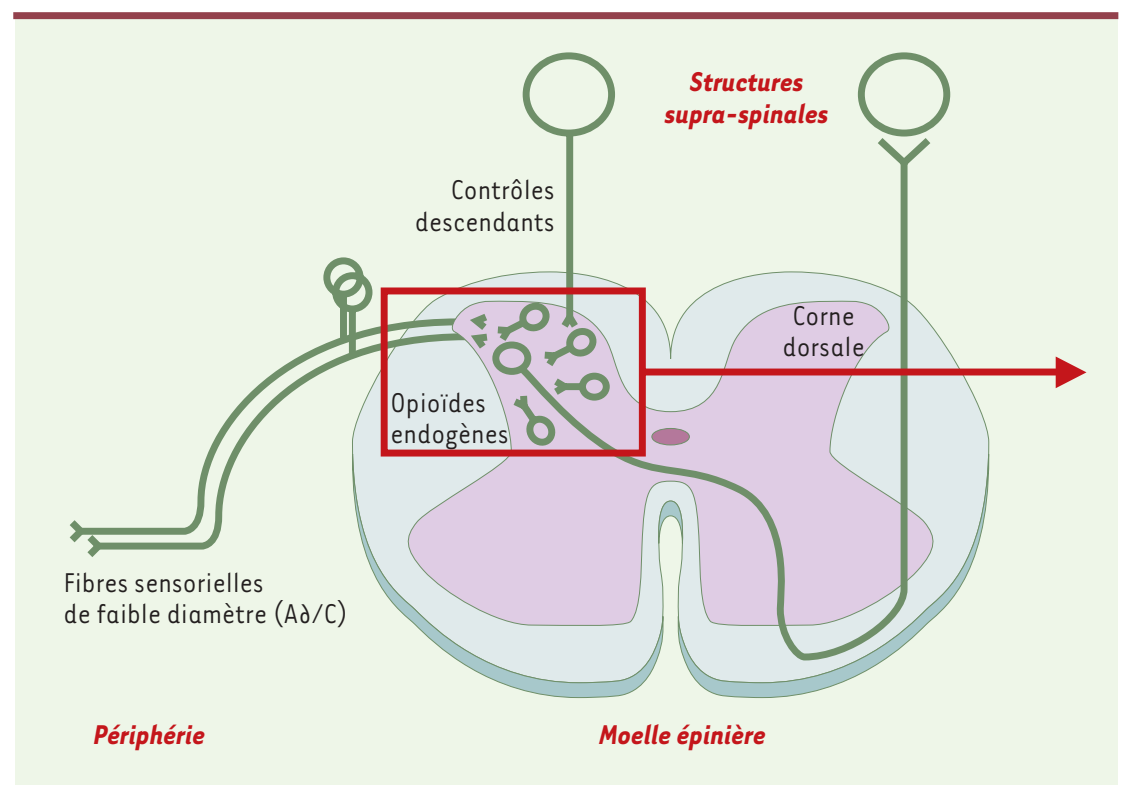

Figure 1. Schéma des relais de la couleur. Les informations « nociceptives», transmises vers leur premier relais central, la corne dorsale de la moelle épinière, par des neurones sensoriels dont l'axone de faible diamètre est peu (Aみ) ou pas myélinisé (C), sont intégrées dans le cortex cérébral pour devenir « douloureuses ». L'efficacité de la transmission spinale est contrôlée, entre autres, par des neurones « descendants » et des interneurones spinaux, mettant en jeu de très nombreux neuromédiateurs au sein desquels les opiödes endogènes jouent un rôle majeur. L'encadré renvoie à la Figure 2. 


\section{RÉFÉRENCES}

1. Cesselin F. Endomorphines, récepteurs des opioïdes et nociception. In : Brasseur L, Chauvin M, Guilbaud G, eds. Douleurs, bases fondamentales, pharmacologie, douleurs aiguës, douleurs chroniques, thérapeutiques. Paris: Maloine, $1997: 65-76$

2. Zadina JE, Hackler L, Ge LJ, Kastin AJ. A potent and selective endogenous agonist for the $\mu$-opiate receptor. Nature 1997 ; $386: 499-502$.
3. Cesselin F, Benoliel J), Bourgoin S, Collin E, Pohl M, Hamon M. Spinal mechanisms of opioid analgesia. In : Stein C, ed. Opioids in pain control: basic and clinical aspects, Cambridge : Cambridge University Press, 1999 : 70-95.

4. Carrion AM, Mellström B, Naranjo JR. Protein kinase A-dependent derepression of the human prodynorphin gene via differential binding to an intragenic silencer element. Mol Cell Biol 1998 ; 18 : 6921-9.
5. Carrion AM, Link WA, Ledo F, Mellström B, Naranjo JR. DREAM is a $\mathrm{Ca}^{2+}$-regulated transcriptional repressor. Nature 1999 ; 398 : 80-4.

6. Cheng HYM, Pitcher GM, Laviolette SR, et al. DREAM is a critical transcriptional repressor for pain modulation. Cell $2002 ; 108$ : $31-43$.

7. Dhawan BN, Cesselin F, Raghubir R, et al. International Union of Pharmacology. XII. Classification of opioid receptors. Pharmacol Rev 1996 ; 48 : 567-92.

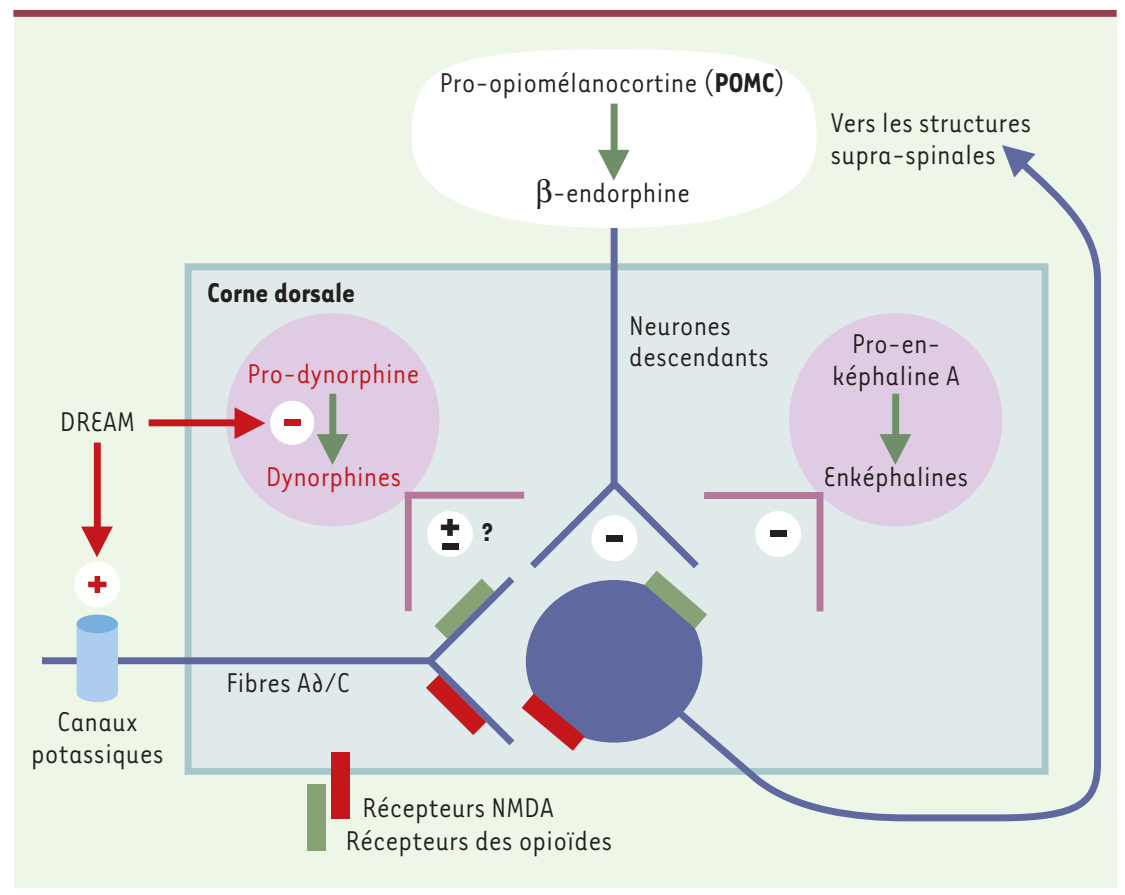

Figure 2. Régulation de la transmission des messages nociceptifs. Les enképhalines (dérivées de la pro-enképhaline $A$ ), synthétisées dans des interneurones spinaux et, dans une bien moindre mesure, la $\beta$-endorphine (issue de la pro-opiomélanocortine : POMC), présente dans les terminaisons spinales de neurones descendants, inhibent la transmission des messages nociceptifs en se liant aux récepteurs des opioïdes. En revanche, les dynorphines (provenant de la prodynorphine), localisées dans des interneurones spinaux, la facilitent, en sollicitant, en outre, les récepteurs $\mathrm{N}$-méthyl-D-aspartate (NMDA) des acides aminés excitateurs. L'article de Cheng et al. [6] va à l'encontre de deux des notions énoncées ci-dessus. Premièrement, ces auteurs observent de nombreux neurones spinaux exprimant le gène codant pour la POMC. Deuxièmement, la réduction de la douleur à la suite de la surexpression spinale des dynorphines (consécutive à l'inactivation du gène codant pour un facteur appelé DREAM, qui normalement réprime l'expression du gène codant pour la pro-dynorphine) laisse penser que les dynorphines exercent une fonction opposée à celle qui leur est communément attribuée. En fait, les observations rapportées par Cheng et al. [6] pourraient être liées à une autre propriété de DREAM : son interaction avec des canaux potassiques des neurones sensoriels (voir texte).

8. Naranjo JR, Mellström B, Acaval M, Sassone-Corsi P. Molecular pathways of pain: Fos/Jun-mediated activation of a noncanonical AP-1 site in the prodynorphin gene. Neuron $1991 ; 6$ : 607-17.

9. Tsou K, Khachaturian $\mathrm{H}$, Akil H, Watson SJ. Immunocytochemical localization of proopiomelanocortin-derived peptides in the adult rat spinal cord. Brain Res 1986 ; 378 : 28-35.

10. Laughlin TM, Vanderah TW, Lashbrook J, et al. Spinally administered dynorphin $\mathrm{A}$ produces long-lasting allodynia: involvement of NMDA but not opioid receptors. Pain 1997 ; 72 : 253-60.

11. Wang Z, Gardell LR, Ossipov MH, et al. Pronociceptive actions of dynorphin maintain chronic neuropathic pain.

J Neurosci 2001 ; 21 : 1779-86.

12. Gerlai R. Gene-targeting studies of mammalian behavior: is it the mutation or the background genotype? Trends Neurosci 1996 ; 19 : 177-81.

13. Buxbaum JD, Choi EK, Luo Y, et al. Calsenilin: a calcium-binding protein that interacts with the presenilins and regulates the levels of a presinilin fragment. Nat Med 1998 ; 10 : 1177-81.

14. An WF, Bowlby MR, Betty M, et al. Modulation of A-type potassium channels by a family of calcium sensors. Nature 2000 ; 403 : 553-6.

15. Fedula SA, Vasiliyev DV, Veselovsky NS. Voltageoperated potassium currents in the somatic membrane of rat dorsal root ganglion neurons: ontogenic aspects. Neuroscience 1998 ; 85 : 497-508.

16. Spreafico F, Barski JJ, Farina C, Meyer M. Mouse DREAM/calselinin/KChIP3: gene structure, coding potential, and expression. Mol Cell Neurosci 2001 ; 17 : 1-16. 